\title{
Midline sensory nerve supply to the anoscrotal junction: a study using human male fetuses
}

\author{
By
}

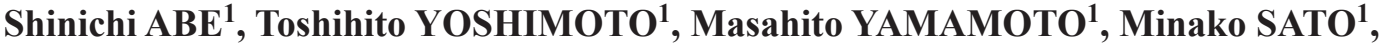 \\ Nobuaki YANAGISAWA ${ }^{2}$, Nobuyuki HINATA ${ }^{3}$, Hiroshi ABE $^{4}$, Murakami GEN ${ }^{1,5}$ \\ ${ }^{1}$ Department of Anatomy, Tokyo Dental College, 2-9-18 Misaki-cho, Chiyoda-ku, Tokyo 101-0061, Japan \\ ${ }^{2}$ Division of Oral Health Sciences, Department of Health Sciences, \\ School of Health and Socialservices Saitama Prefectural University \\ ${ }^{3}$ Department of Urology, Kobe University Graduate School of Medicine, 7-5-1, Kusunoki-cho, Kobe 650-0017, Japan \\ ${ }^{4}$ Department of Anatomy, Akita University School of Medicine, 1-1-1 Hondo, Akita 010-8543, Japan \\ ${ }^{5}$ Division of Internal Medicine, Iwamizawa Asuka Hospital, 297 Shibun cho, Iwamizawa 068-0833, Japan
}

- Received for Publication, February 16, 2017-

Key Words: Skin nerve, scrotum, pelvic nerve plexus, perineal raphe, human fetus

\begin{abstract}
Summary: The origin of the posterior scrotal nerve is considered to be the bilateral pudendal nerves but the course to the midline is still obscure. Using 5 late-stage human male fetuses, we identified the single nerve through the intramuscular midline septum of the bulbospongiosus and the bilateral nerves along the left and right sides of the septum. Thus, the posterior scrotal nerve showed a variation: a single midline trunk or bilateral nerves. Branches of the bilateral pudendal nerves ran medially between the muscle and Cowper's gland and, at the midline area, they joined or associated closely. During the proximal course, much or less, the nerve penetrated the superior part of the muscle. The nerve entered the subcutaneous tissue at and near the perineal raphe. The communication with intrapelvic autonomic nerves were suggested behind Cowper's gland. Notably, the midline skin immediately anterior to the anus carried a considerable dense supply of thin sensory nerves. However, these nerves seemed to come from a space between the rectal smooth muscle and the external anal sphincter, not from the posterior scrotal nerve. Therefore, surgical treatment of the intersphincteric layer was likely to injure the original sensory nerve supply to the anterior anal skin.
\end{abstract}

\section{Introduction}

Nerves of the anoscrotal junction has been one of major interests of researchers because they should contain an afferent route for autonomic nerve reflexes in the pelvic floor involving the urinary and anal sphincter functions (Bird and Hanno, 1998; Lefaucheur, 2006; Yilmaz et al., 2009; Hotta et al., 2012). The posterior part of the human scrotum is innervated by a branch of the pudendal nerve, i.e., the posterior scrotal nerve. From a surgical implication, Yucel and Baskin (2003) noted a fact that the posterior scrotal nerve runs though the perineal raphe (a subcutaneous, midline structure of the scrotum). However, these researchers did not demonstrate the nerve course from the laterally-located perineal nerve to the midline area. Although she applied a term "ventral nerve of the penis", Takahashi (1980) described that a part of the perineal nerves from the pudendal nerve passes through the central tendinous point of the perineum and, therein, it communicates with intrapelvic autonomic nerve branches. Because of the close topographical relation to the bulbospongiosus muscle in the nerve course, we considered her ventral nerve of the penis corresponding to the posterior scrotal nerve. To our regret, all of the adult cadaveric specimens seemed to be bisected along the midline before her minute dissection.

The urorectal septum of endodermal origin plays a role of zipper to close the bilateral genital folds to product the perineal raphe (Seifert et al., 2008; Georgas et al., 2015). The raphe is a superficial continuation of the midline septum between the bilateral bulbospongiosus muscles (Jin et al., 2016). Because the intramuscular septum is very tight in histology, in the proximal course before entering the subcutaneous tissue, we speculated that the posterior scrotal nerve did not run through the tight intermuscular septum but along the lateral aspects 
of the bulbospongiosus or through the muscle. A midline skin nerve is rare in the human body. The aim of this study was to ensure the origin and course of the posterior scrotal nerve using histological sections of the late-stage human fetuses.

\section{Materials and Methods}

The study was performed in accordance with the provisions of the Declaration of Helsinki 1995 (as revised in 2013). We used frontal sections of the pelvic floor obtained from 5 human male fetuses at 24-33 weeks (crown-rump length [CRL], 185-265 mm). These fetuses were parts of a collection in Department of Anatomy, Akita University, Akita, Japan. They were donated by their families to the Department during 1975-1985 and preserved in $10 \% \mathrm{w} / \mathrm{w}$ neutral formalin solution for more than 30 years. The available data was limited to the date of donation and the gestational weeks, but we did not find a document saying the family name, the name of obstetricians or hospital and the reason of abortion. The use for research was approved by the university ethics committee in Akita (No. 1378). For sectioning, the specimens were decalcified by incubating them at room temperature in Plank-Rychlo solution $\left(\mathrm{AlCl}_{2} / 6 \mathrm{H}_{2} \mathrm{O}, 7.0 \mathrm{w} / \mathrm{v} \% ; \mathrm{HCl}, 3.6\right.$; $\mathrm{HCOOH}, 4.6)$ for $1-2$ weeks.

After routine procedure for paraffin embedded histology, we prepared semiserial frontal sections (7-10 micron thickness) with 50-100 micron interval. Since sections from 2 of the 5 specimens were available for immunohistochemistry of S100 protein, every 10th section was used for the nerve staining in spite of the severe condition of decalcification. The primary antibody was mouse monoclonal anti-human S100B protein (Dako N1573; Dako, Glostrup, Denmark; dilution 1:100). Antigen retrieval was not performed. The secondary antibody (Dako ChemMate Envision Kit, Dako, Glostrup, Denmark) was labeled with horseradish peroxidase (HRP), and antigen-antibody reactions were detected by the HRP-catalyzed reaction with diaminobenzidine. Counterstaining with hematoxylin was performed on the same samples. Observations and taking photographs were usually performed with Nikon Eclipse 80 , but photos at ultra-low magnification (less than $\mathrm{x} 1$ at the objective lens) were taken using a high grade flat scanner (Epson GTX970) with translucent illumination.

\section{Results}

The anoscrotal junction area was likely to include both of the external anal sphincter and the bulbospongiosus muscle in frontal planes (Figs. 1-6). In all 5 specimens, the intramuscular midline septum was evident between the bilateral bulbospongiosus muscles (Figs. 2A, 4A and $5 \mathrm{~B})$ and it continued superficially to the subcutaneous perineal raphe (Figs. 4A and 5C) and deeply to the midline septum of the corpus cavernosum penis (Fig. 5A). We were able to identify a single nerve through the tight midline septum in 2 specimens (Fig. 2AB). However, in the other 3 specimens, the bilateral posterior scrotal nerves were also found running along the left and right sides of the muscular midline septum (Fig. 4BC and Fig. 5D). Therefore, the posterior scrotal nerve showed a variation: a single midline trunk or bilateral nerves. However, the single nerve should contain the bilateral origins.

Branches of the bilateral pudendal nerves ran medially between the bulbospongiosus and Cowper's gland (Fig. 2DE) and, at the midline area, they joined to provide a single nerve or associated closely. During the proximal course to the midline, much or less, the nerve penetrated the superior part of the muscle (Fig. 4D). The nerve entered the subcutaneous tissue at and near the perineal raphe (Figs. 4B and 5C). In contrast to the superior and internal aspects of the bulbospongiosus muscle, no or few skin nerves ran through a wide, lateral fascial space between the bulbospongiosus and ischiocavernosus muscles.

In a specimen at 30 weeks (Figs. 5 and 6), the midline nerves were connected with a nerve plexus behind Cowper's gland and below the rhabdosphincter. Most of composite nerves of the plexus came from the pelvic plexus branches running along the lateral aspects of the bladder and prostate (Fig. 6DE). In the same specimen, a few Pacinian corpuscles was found in the ischiocavernous fossa (Fig. 5GH).

The dense innervation was evident in the midline skin immediately anterior to the anus (Fig $3 \mathrm{~A}-\mathrm{D}$ ) as well as beneath the stratified squamous epithelium in the anal canal (Fig. 3E). These thin nerves ran along the postero-anterior axis and they appeared to come from nerves passing between the external anal sphincter and the circular smooth muscle of the rectum, not from the posterior scrotal nerve. Thus, the nerve density appeared to be higher in the more posterior area although we did not measure the difference.

\section{Discussion}

In the beginning of this study, we were interested in how the bilateral pudendal nerves provide a single posterior scrotal nerve along the midline. However, there was a variation; a single midline trunk or bilateral nerves. Rather than the subcutaneous raphe, the nerve course was characterized by its close relation to the tight intermuscular septum of the bilateral bulbospongiosus muscles. In male fetuses, the muscle is attached to or even intermingled with the external anal sphincter (Arakawa et al., 2010, 2015). The developing subcutaneous raphe is connected with and reinforced by the intermusclular septum in midterm fetuses (Jin et al., 2016). The posterior scrotal nerve seemed to develop earlier than the raphe 

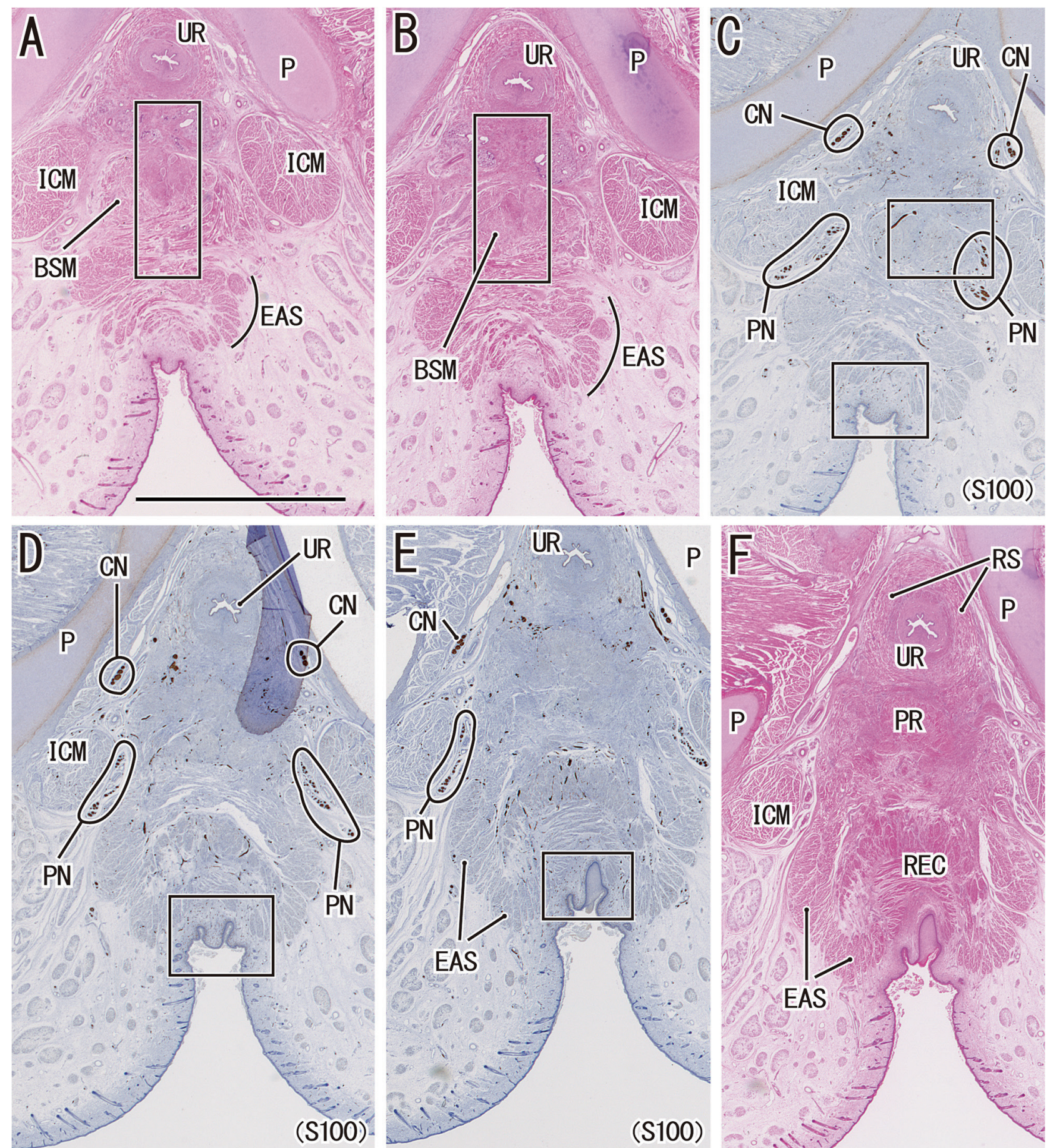

Fig. 1. Topographical anatomy of the perineal area immediately anterior to the anus.

Frontal sections of a specimen at 33 weeks (CRL $265 \mathrm{~mm}$ ). HE staining (panels A, B and F) and immunohistochemistry of S100 protein (panels $\mathrm{C}-\mathrm{E})$. Panel A (Panel F) shows the most anterior (posterior) site in the figure. Intervals between panels are $0.5 \mathrm{~mm}(\mathrm{~A}-\mathrm{B}, \mathrm{B}-\mathrm{C}, \mathrm{C}-\mathrm{D}, \mathrm{D}-\mathrm{E})$ and $0.05 \mathrm{~mm}(\mathrm{E}-\mathrm{F})$. The anterior part of the external anal sphincter (EAS) is located below the bulbospongiosus muscle (BSM) in panels A-C. The circular smooth muscle of the rectum (REC) is seen in panels $\mathrm{E}$ and $\mathrm{F}$. In panels $\mathrm{C}-\mathrm{E}$, the pudendal nerve (PN) is distant from the cavernous nerve (CN). Squares including the skin in panels C, D and E will be shown in Fig. 3, while the other squares including the deep tissue in panels A-C will be shown in Fig. 2. All panels are prepared at the same magnification (scale bar in panel A, 5 mm). Other abbreviations, see the common abbreviation for figures. 

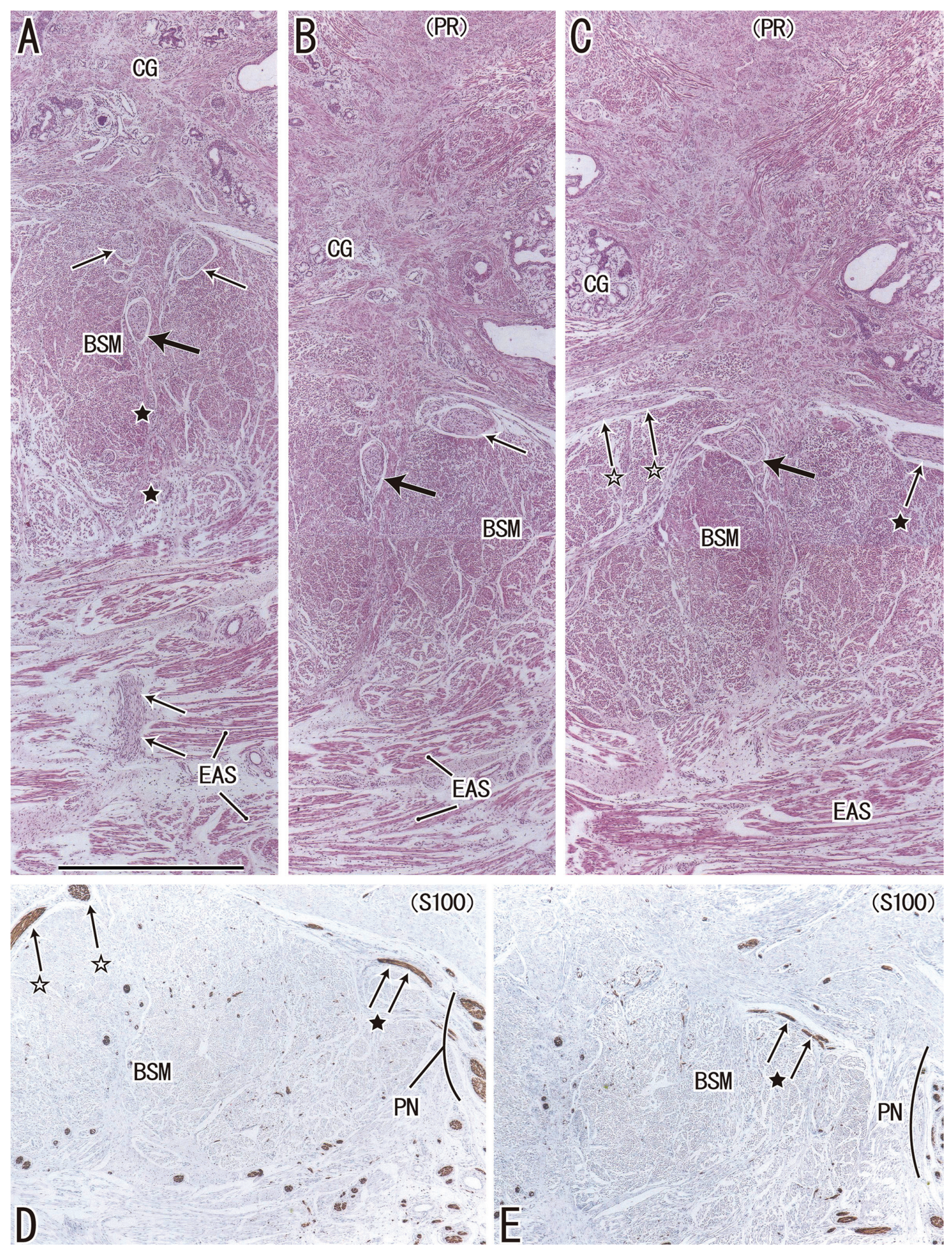

Fig. 2. Posterior scrotal nerve through the midline septum between the bilateral bulbospongiosus muscles.

The same specimen as shown in Fig. 1 (33 weeks; CRL $265 \mathrm{~mm}$ ). HE staining (panels A-C) and immunohistochemistry of S100 protein (panels D and E). Panel A (Panel E) shows the most anterior (posterior) site in the figure. Intervals between panels are $0.2 \mathrm{~mm}(\mathrm{~A}-\mathrm{B}), 0.3$ $\mathrm{mm}(\mathrm{B}-\mathrm{C})$ and $0.5 \mathrm{~mm}(\mathrm{C}-\mathrm{D}, \mathrm{D}-\mathrm{E})$. Panels $\mathrm{A}, \mathrm{C}$ and $\mathrm{D}$ are higher magnification views of squares in Fig. 1A-C, respectively. In panel A, bilateral nerves (thin arrows) appear to join to form a single midline nerve (thick arrow) through the midline septum (stars) of the bulbospongiosus muscle (BSM). The other thin arrows indicate a skin nerve near the external anal sphincter (EAS). Thin arrow in panel B indicates the unilateral nerve origin. Panel C exhibits the bilateral origins (open stars and black star) of the midline nerve (thick arrow). Panels D and E display the proximal course of the nerve (open stars and black star) coming from the pudendal nerve (PN). All panels are prepared at the same magnification (scale bar in panel A, $1 \mathrm{~mm}$ ). Other abbreviations, see the common abbreviation for figures. 

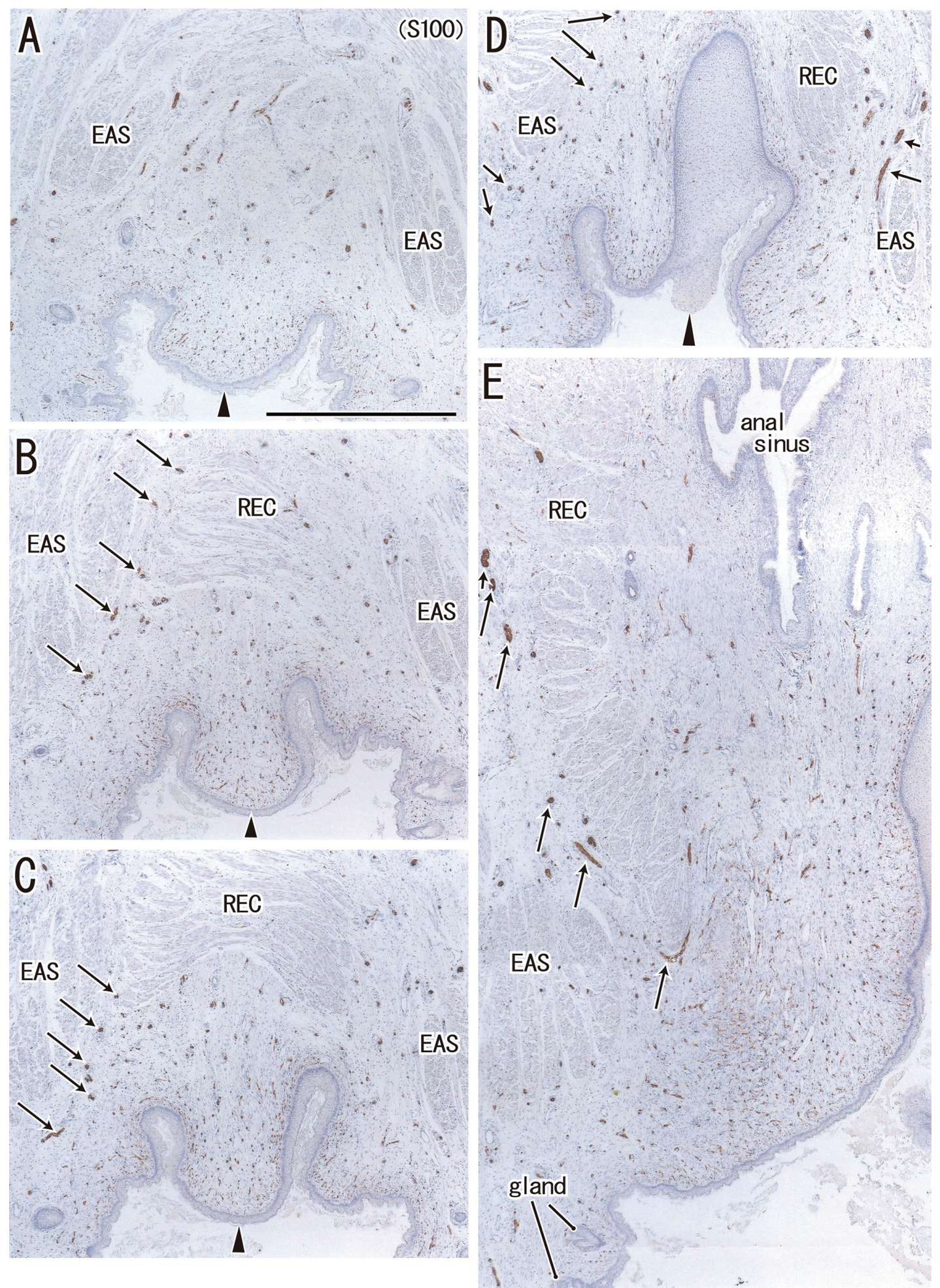

Fig. 3. Thin nerves in the midline anal skin.

The same specimen as shown in Fig. 1 (33 weeks; CRL 265 mm). Immunohistochemistry of S100 protein. Panel A (Panel E) shows the most anterior (posterior) site in the figure. Intervals between panels are $0.3 \mathrm{~mm}(\mathrm{~A}-\mathrm{B}), 0.2 \mathrm{~mm}(\mathrm{~B}-\mathrm{C}), 0.5 \mathrm{~mm}(\mathrm{C}-\mathrm{D})$ and $1.5 \mathrm{~mm}(\mathrm{D}-\mathrm{E})$. Panels $\mathrm{A}, \mathrm{C}$ and $\mathrm{D}$ are higher magnification views of squares in Fig. $1 \mathrm{C}-\mathrm{E}$, respectively. Arrowhead in panels $\mathrm{A}-\mathrm{D}$ indicate the midline. The dense innervation is evident in the midline skin immediately anterior to the anus (panels A-D) as well as in the skin area with stratified squamous epithelium in the anal canal (panel E). These thin nerves appeared to come from nerves passing between the external anal sphincter (EAS) and the circular smooth muscle of the rectum (REC). All panels are prepared at the same magnification (scale bar in panel A, $1 \mathrm{~mm}$ ). Other abbreviations, see the common abbreviation for figures. 

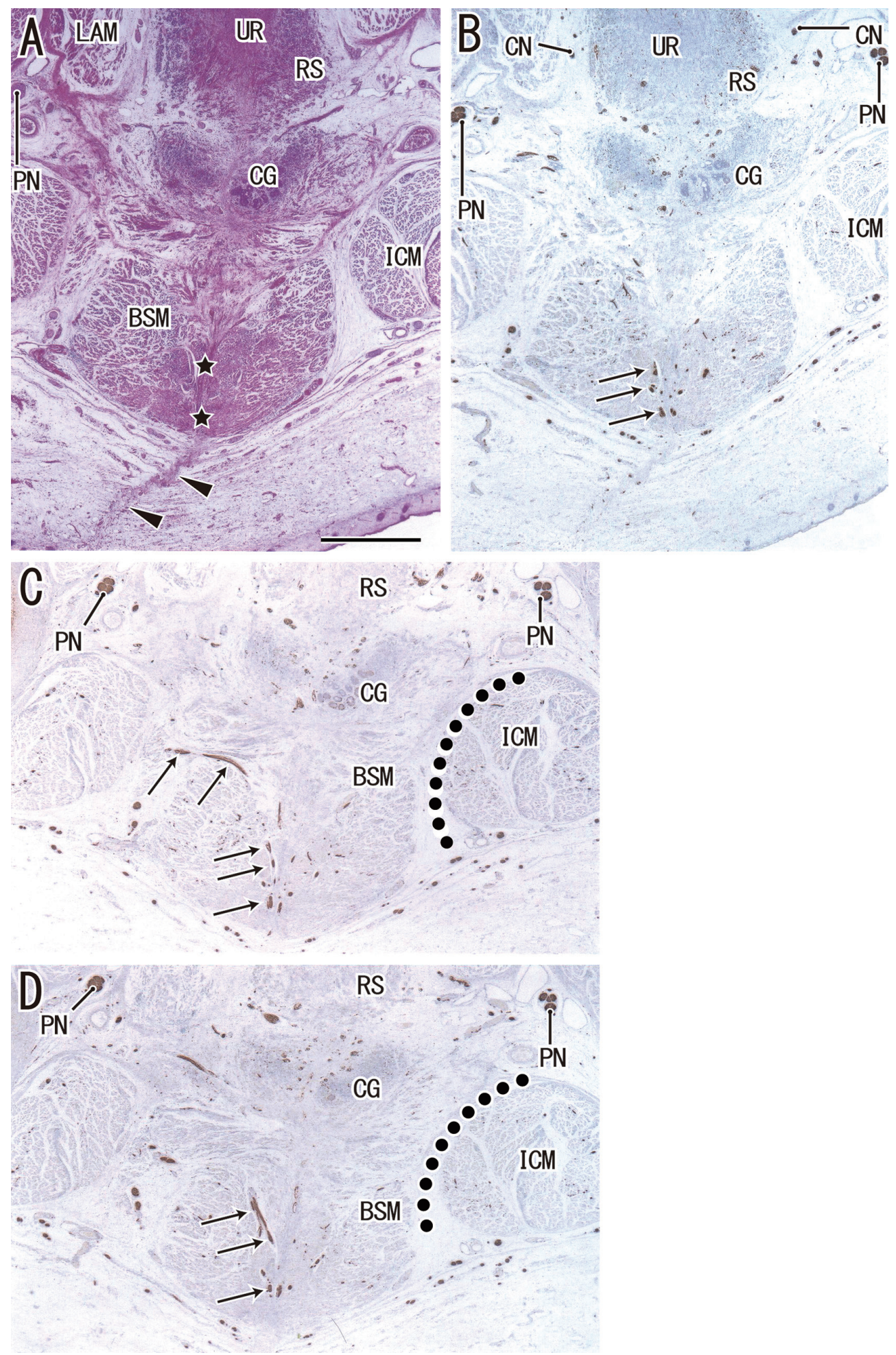

Fig. 4. Posterior scrotal nerve along the midline septum between the bilateral bulbospongiosus muscles.

Frontal sections of a specimen at 24 weeks (CRL $180 \mathrm{~mm}$ ). HE staining (panel A) and immunohistochemistry of S100 protein (panels B-D). Panel A (Panel D) shows the most anterior (posterior) site in the figure. Intervals between panels are $0.1 \mathrm{~mm}(\mathrm{~A}-\mathrm{B})$ and $0.2 \mathrm{~mm}(\mathrm{~B}-\mathrm{C}, \mathrm{C}-\mathrm{D})$. In panel A, black stars indicate the midline septum between the bilateral bulbospongiosus muscles (BSM) and it continues to the subcutaneous perineal raphe (arrowheads). The bilateral posterior scrotal nerves (arrows) are seen along the midline septum of the muscle and they appear to come from the lateral side of the muscle (panel C). No nerve runs through a wide fascial space between the bulbospongiosus and ischiocavernosus muscles (dotted line). All panels are prepared at the same magnification (scale bar in panel A, $1 \mathrm{~mm}$ ). Other abbreviations, see the common abbreviation for figures. 


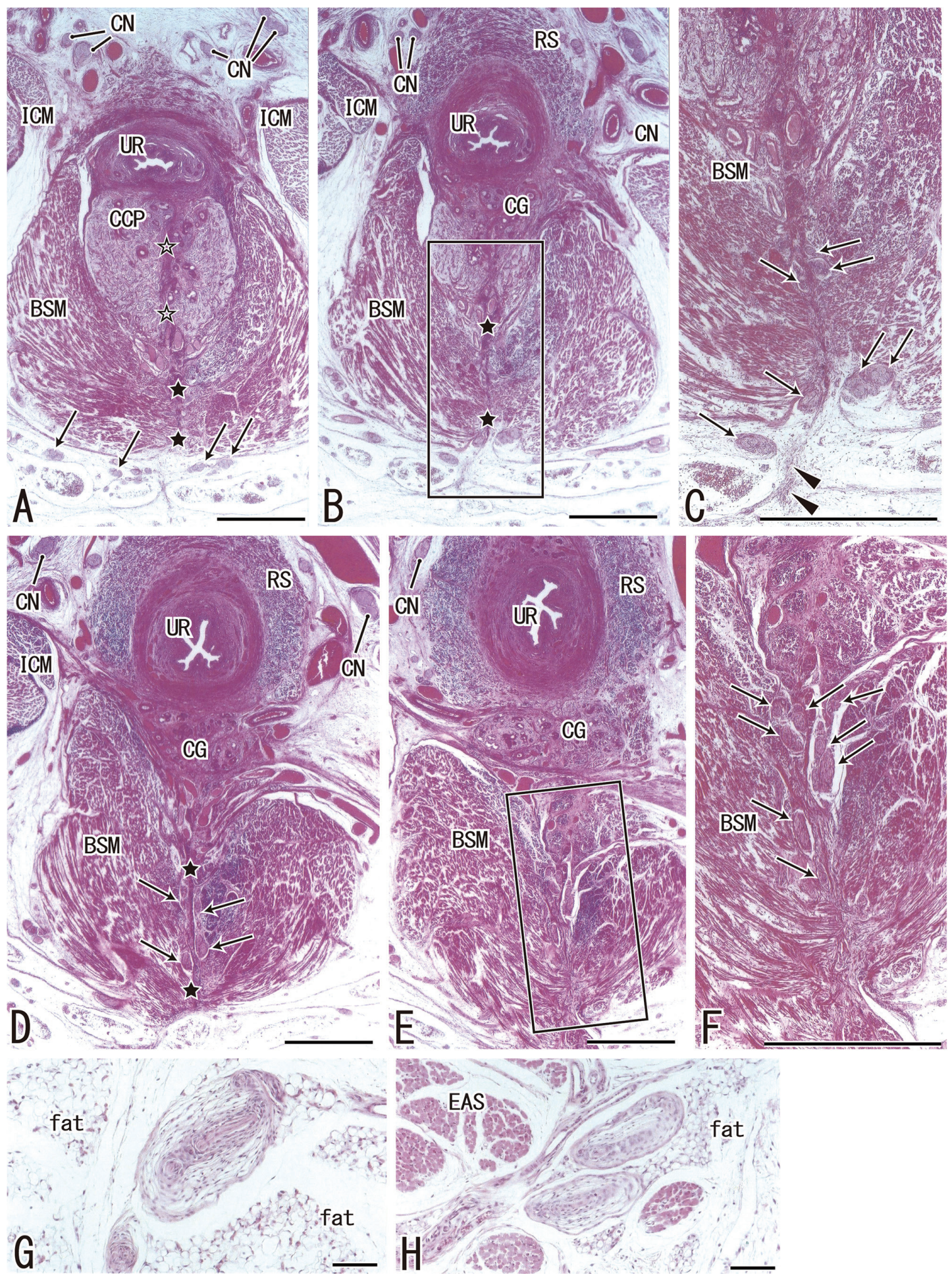

Fig. 5. Posterior scrotal nerve along the midline septum between the bilateral bulbospongiosus muscles.

Frontal sections of a specimen at 30 weeks (CRL $255 \mathrm{~mm}$ ). HE staining. Panel A (Panel E) shows the most anterior (posterior) site in the figure. Panels $\mathrm{C}$ and $\mathrm{F}$ are higher magnification views of a square in panel $\mathrm{B}$ or $\mathrm{E}$, respectively. Intervals between panels are $0.8 \mathrm{~mm}(\mathrm{~A}-\mathrm{B})$ and $0.4 \mathrm{~mm}(\mathrm{~B}-\mathrm{D}, \mathrm{D}-\mathrm{E})$ Open stars indicate the midline septum of the corpus cavernosum penis (CCP), while black stars indicate the midline septum between the bilateral bulbospongiosus muscles (BSM). The posterior scrotal nerve (arrows) enters the subcutaneous tissue in panel A and runs through the muscle septum in panels C and F. Not a single nerve but bilateral nerves are identified in the left and right sides of the septum (panel D). The muscle septum continues to the subcutaneous perineal raphe (arrowheads) in panel C. In the same specimen, Pacinian corpuscles are seen in the ischiocavernous fossa (panels $\mathrm{G}$ and $\mathrm{H}$ ). Scale bars, $1 \mathrm{~mm}$. Other abbreviations, see the common abbreviation for figures. 

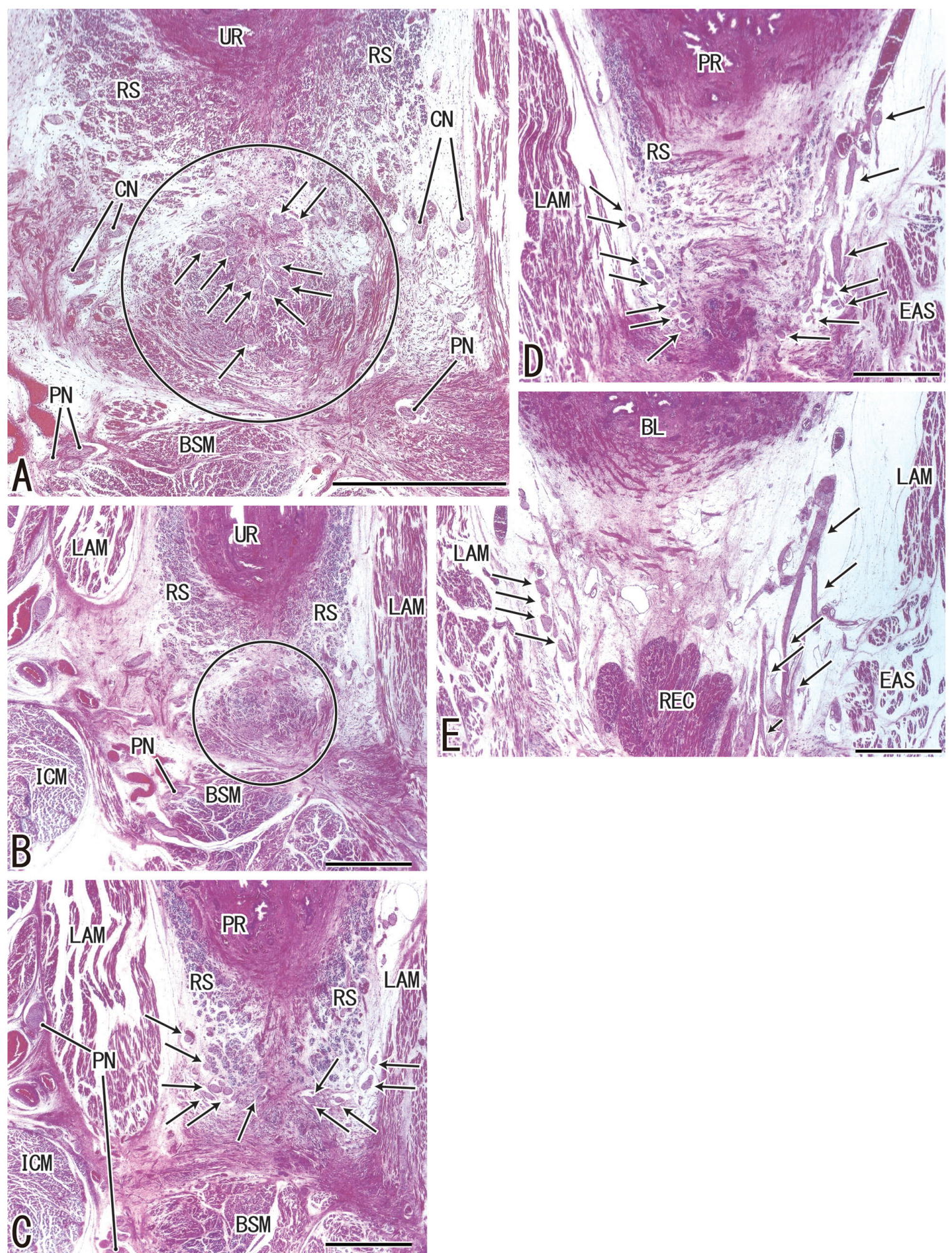

Fig. 6. A nerve plexus behind Cowper's gland: an origin of the midline skin nerve.

The same specimen as shown in Fig. 5. Frontal sections. HE staining. Panel A (Panel E) shows the most anterior (posterior) site in the figure. Panel A is located $0.8 \mathrm{~mm}$ posterior to Fig. 1EF. Panel A is a higher magnification views of panel B: the circle in panel A corresponds to that in panel B. Intervals between panels are $0.4 \mathrm{~mm}(\mathrm{~B}-\mathrm{C}, \mathrm{C}-\mathrm{D}, \mathrm{D}-\mathrm{E})$. Behind Cowper's gland (CG) and below the rhabdosphincter (RS), a nerve plexus (arrows in panel A) is seen and it is connected with the midline nerves shown in Fig. 5. Most of composite nerves of the plexus come from the pelvic plexus branches running along the lateral aspects of the bladder and prostate (arrows in panels $\mathrm{C}-\mathrm{E}$ ). Scale bars, $1 \mathrm{~mm}$. Other abbreviations, see the common abbreviation for figures. 
and septum and, later it might be involved into them. Irrespective of whether the nerve was a trunk or bilateral, the proximal course was always located just below Cowper's gland: this site seemed to correspond to the so-called cavernous nerve mesh embedded in the adult recto-urethralis muscle behind the membranous urethra (Hinata et al., 2015). Actually, in the present study, a limited 1 specimen strongly suggested a connection between the midline skin nerve and the pelvic plexus branches. Some of sensory nerve fibers of the posterior scrotal nerve were likely to reach the spinal dorsal root ganglion via the intrapelvic course such as the pelvic splanchnic nerves.

Yucel and Baskin (2003) noted the midline course of the posterior scrotal nerve in view of kinds of plastic and reconstructive surgery. Notably, rather than the scrotum, the midline skin immediately anterior to the anus carried a considerably dense supply of thin sensory nerves. Moreover, these nerves seemed to come from a space between the rectal smooth muscle and the external anal sphincter (i.e., the intersphincteric layer), not from the posterior scrotal nerve. A major origin of intersphincteric nerves is not the pudendal nerve but the pelvic nerve plexus and/ or the myenteric plexus of the rectum (Hieda et al., 2013; Ishiyama et al., 2014). Anal skin touch was likely to connect with autonomic nerve reflexes involving evacuation. Surgical treatment of the intersphincteric layer, that is so commonly performed in recent cancer surgery, was likely to injure the sensory nerve supply to the anterior anal skin. After injury, the original nerves might be replaced by an expanding area of innervation from the posterior scrotal nerve. In addition, as shown in the present figure, Li et al. (1992) found Pacinian corpuscle in the human pelvic floor using silver impregnation method.

\section{References}

1) Bird SJ, Hanno PM: Bulbocavernous reflex studies and autonomic testing in the diagnosis of erectile dysfunction. J Neurol Sci 1998; 154:8-13

2) Lefaucheur JP: Neurophysiological testing in anorectal disorders. Muscle Nerve 2006; 33:324-333.

3) Yilmaz U, Vicars B, Yang CC: Evoked cavernous activity: neuroanatomic implications. Int J Impot Res 2009; 21:301-305.

4) Hotta H, Masunaga K, Miyazaki S, Watanabe N, Kasuya Y: A genital mechanical skin stimulation technique for inhibition of micturition contractions of the urinary bladder. Auto Neurosci 2012; 167:12-20.

5) Yucel S, Baskin LS: The neuroanatomy of the human scrotum: surgical ramifications. Br J Urol Int 2003; 91:393-397.

6) Takahashi M: Morphological analysis of the pudendal nerve. Acta Anat Nippon 1980; 55:23-44.

7) Seifert AW, Harfe BD, Cohn MJ: Cell lineage analysis demon- strates an endodermal origin of the distal urethra and perineum. Dev Biol 2008; 318:143-152.

8) Georgas KM, Armstrong J, Keast JR, Larkins CE, McHugh KM, Southard-Smith EM, Cohn MJ, Batourina E, Dan H, Schneider K, Buehler DP, Wiese CB, Brennan J, Davies JA, Harding SD, Baldock RA, Little MH, Vezina CM, Mendelsohn C: An illustrated anatomical ontology of the developing mouse lower urogenital tract. Development 2015; 142:1893-1908.

9) Jin ZW, Jin Y, Li XW, Murakami G, Rodríguez-Vázquez JF, Wilting J: Perineal raphe with special reference to its connection to perineal muscles: a histological study using human fetuses. Anat Cell Biol 2016 (in press).

10) Arakawa T, Hayashi S, Kinugasa Y, Murakami G, Fujimiya M: Development of the external anal sphincter with special reference to intergender difference: observations of mid-term fetuses (15-30 weeks of gestation) Okajimas Folia Anat Jpn 2010; 87:49-58.

11) Arakawa $\mathrm{T}, \mathrm{Yu} \mathrm{HC}$, Kim JH, Joerg W, Rodriguez-Vazquez JF, Murakami G, Cho BH: Fetal growth of the anal sinus and sphincters with special reference to a hypothetical role of the growing sinus to avoid anal canal stenosis. Int J Colorectal Dis 2015; 31: 493-502.

12) Hinata N, Murakami G, Miyake H, Abe S, Fujisawa M: Histological study of the cavernous nerve mesh outside the periprostatic region: anatomical basis for erectile function after non-nervesparing radical prostatectomy. J Urol 2015; 193:1052-1059.

13) Hieda K, Cho KH, Arakawa T, Fujimiya M, Murakami G, Matsubara M: Nerves in the intersphincteric space of the human anal canal with special reference to their continuation to the enteric nerve plexus of the rectum. Clin Anat 2013; 26:843-854.

14) Ishiyama G, Hinata N, Kinugasa $Y$, Hieda K, Murakami G, Fujimiya F: Nerves to the internal anal sphincter: an immunohistochemical study using donated elderly cadavers. Surg Radiol Anat 2014; 36: 1033-1042.

15) Li L, Li Z, Huo HS, Wang HZ, Wang LY: Sensory nerve endings in the puborectalis and anal region of the fetus and newborn. Dis Colon rectum 1992; 35:552-559.

\section{Figure legends}

Common abbreviation for figures:

BL, urinary bladder;

BSM, bulbospongiosus muscle;

$\mathrm{CCP}$, corpus cavernosum penis;

CG, Cowper's gland;

$\mathrm{CN}$, cavernous nerve candidate;

EAS, external anal sphincter;

ICM, ischiocavernosus muscle;

LAM, levator ani muscle;

$\mathrm{PN}$, pudendal nerve;

PR, prostate;

REC, rectal smooth muscles;

RS, rhabdosphincter of the urethra;

UR, urethra. 